\title{
ON A THEOREM OF BISHOP AND COMMUTANTS OF TOEPLITZ OPERATORS IN $\mathbb{C}^{n}$
}

\author{
SÖNMEZ ŞAHUTOĞLU AND AKAKI TIKARADZE
}

\begin{abstract}
We prove an approximation theorem on a class of domains in $\mathbb{C}^{n}$ on which the $\bar{\partial}$ problem is solvable in $L^{\infty}$. Furthermore, as a corollary, we obtain a version of the Axler-ČučkovićRao Theorem in higher dimensions.
\end{abstract}

Let $\Omega$ be a domain in $\mathbb{C}^{n}$ and $\phi$ be a complex-valued function on $\Omega$. Let $H^{\infty}(\Omega)$ and $H^{\infty}(\Omega)[\phi]$ denote the set of bounded holomorphic functions on $\Omega$ and the algebra generated by $\phi$ over $H^{\infty}(\Omega)$, respectively. In 1989, Christopher Bishop proved the following approximation theorem (see [Bis89, Theorem 1.2]).

Theorem (Bishop). Let $\Omega$ be an open set in $\mathbb{C}$ and $f$ be a bounded holomorphic function on $\Omega$ that is non-constant on every connected component of $\Omega$. Then $H^{\infty}(\Omega)[\bar{f}]$ is dense in $C(\bar{\Omega})$ in the uniform topology.

In the same paper, Christophe Bishop also proved a stronger approximation result, [Bis89, Theorem 1.1], on a more restrictive class of domains on which $\bar{f}$ is only assumed to be a nonholomorphic harmonic function. Such a result for the unit disc goes back to Sheldon Axler and Allen Shields [AS87]. Recently, Guangfu Cao gave an incorrect statement [Cao08, Theorem 5] in an attempt to give a higher dimensional version of Bishop's Theorem. Alexander Izzo and Bo Li [IL13, pg 246] noticed that the statement is incorrect. Håkan Samuelsson and Erlend Wold in [SW12, Theorem 1.3] proved a partial extension of Bishop's Theorem for pluriharmonic functions and $C^{1}$-smooth polynomially convex domains in $\mathbb{C}^{n}$.

This article is motivated by these papers and is an attempt to contribute an approximation theorem akin to Bishop's Theorem on domains in $\mathbb{C}^{n}$. We are not able to generalize Bishop's theorem to $\mathbb{C}^{n}$ and this is still an open problem. However, we prove approximation results under some restrictions on the functions and the domains. Furthermore, we apply our results to prove a version of the Axler-Čučković-Rao Theorem [AČR00] in higher dimensions.

To present our first result we need to make some definitions. Let $\Omega \subset \mathbb{C}^{n}$ be a pseudoconvex domain and $C L_{(0, q)}^{\infty}(\Omega)$ denote the set of $(0, q)$-forms with coefficient functions that are

Date: October 15, 2018.

2010 Mathematics Subject Classification. Primary 46J15; Secondary 32A65.

Key words and phrases. Bishop's theorem, pseudoconvex domain, Toeplitz operator.

The second author is supported in part by the University of Toledo Summer Research Awards and Fellowships Program. 
$C^{\infty}$-smooth and bounded on $\Omega$. That is, $C L_{(0, q)}^{\infty}(\Omega)=L_{(0, q)}^{\infty}(\Omega) \cap C_{(0, q)}^{\infty}(\Omega)$. We call $\Omega$ a $L^{\infty}$ pseudoconvex domain if for $1 \leq q \leq n$, and $f \in C L_{(0, q)}^{\infty}(\Omega)$ such that $\bar{\partial} f=0$ there exists $g \in L_{(0, q-1)}^{\infty}(\Omega)$ such that $\bar{\partial} g=f$.

The class of $L^{\infty}$-pseudoconvex domains include the products of $C^{2}$-smooth bounded strongly pseudoconvex domains [SH80], smooth bounded pseudoconvex finite type domains in $\mathbb{C}^{2}$ [Ran90], smooth bounded finite type convex domains in $\mathbb{C}^{n}$ [DFF99], and some infinite type smooth bounded convex domains in $\mathbb{C}^{2}$ [FLZ11].

Given a holomorphic mapping $f: \Omega \rightarrow \mathbb{C}^{m}$ (where $\Omega \subset \mathbb{C}^{n}$ ) and $\lambda \in \mathbb{C}^{m}$, we denote the union of all non-isolated points of $f^{-1}(\lambda)$ by $\Omega_{f, \lambda}$. Since $f^{-1}(\lambda)$ is a complex subvariety of $\Omega$ (for $\lambda$ in the range of $f$ ), it follows that $\Omega_{f, \lambda}$ is the union of all positive dimensional connected components of $f^{-1}(\lambda)$. In the case $f$ extends smoothly up to the boundary of $\Omega$, we define $\Omega_{f, \lambda}^{\prime}$ to be the union of all non-isolated points of $f^{-1}(\lambda)$ within $\bar{\Omega}$. Clearly $\Omega_{f, \lambda}^{\prime} \subset \Omega_{f, \lambda} \cup b \Omega$ where $b \Omega$ denotes the boundary of $\Omega$. We define

$$
\Omega_{f}=\bigcup_{\lambda \in \mathbb{C}^{m}} \Omega_{f, \lambda} .
$$

It is clear that $\Omega_{f}$ is a subset of the set where the Jacobian of $f$ has rank strictly less than $n$.

Now we are ready to present our first approximation result.

Theorem 1. Let $\Omega$ be a bounded $L^{\infty}$-pseudoconvex domain in $\mathbb{C}^{n}$ and $f_{j} \in H^{\infty}(\Omega)$ for $j=1, \ldots, m$. Assume that $g \in C(\bar{\Omega})$ such that $\left.g\right|_{b \Omega \cup \Omega_{f}}=0$ where $f=\left(f_{1}, \ldots, f_{m}\right)$. Then $g$ belongs to the closure of $H^{\infty}(\Omega)\left[\overline{f_{1}}, \cdots, \overline{f_{m}}\right]$ in $L^{\infty}(\Omega)$.

Theorem 11] and [IL13, Theorem 4.2] lead to the following corollary.

Corollary 1. Let $\Omega$ be a bounded $L^{\infty}$-pseudoconvex domain in $\mathbb{C}^{n}$ and $f_{j} \in H^{\infty}(\Omega)$ for $j=1, \ldots, m$ and $n \leq m$. Then the following are equivalent.

i. $H^{\infty}(\Omega)\left[\overline{f_{1}}, \ldots, \overline{f_{m}}\right]$ is dense in $L^{p}(\Omega)$ for all $0<p<\infty$,

ii. $H^{\infty}(\Omega)\left[\overline{f_{1}}, \ldots, \overline{f_{m}}\right]$ is dense in $L^{p}(\Omega)$ for some $1 \leq p<\infty$,

iii. the Jacobian of $f=\left(f_{1}, \ldots, f_{m}\right)$ has rank $n$ for some $z \in \Omega$.

To formulate our next result we will need the following notation. The set of holomorphic functions on $\Omega$ that have smooth extensions up to the boundary is denoted by $A^{\infty}(\Omega)$. Given a compact set $K \subset \bar{\Omega}$, we will denote by $A_{\bar{\Omega}}(K)$ the norm closed subalgebra of continuous functions on $K$ spanned by restrictions of $A^{\infty}(U \cap \Omega)$ onto $K$, where $U$ runs through open neighborhoods of $K$.

Theorem 2. Let $\Omega$ be a smooth bounded pseudoconvex domain in $\mathbb{C}^{n}$ and $f_{j} \in A^{\infty}(\Omega)$ for $j=1, \ldots, m$. Then $g \in C(\bar{\Omega})$ belongs to the closure of $A^{\infty}(\Omega)\left[\overline{f_{1}}, \cdots, \overline{f_{m}}\right]$ in $L^{\infty}(\Omega)$ if and only if for any $\lambda$ in the range of $f=\left(f_{1}, \ldots, f_{m}\right)$ we have $\left.g\right|_{\Omega_{f, \lambda}^{\prime}} \in A_{\bar{\Omega}}\left(\Omega_{f, \lambda}^{\prime}\right)$. 
Alexander Izzo in [Izz11, Theorem 1.3] proved (among other things) the following interesting result.

Theorem (Izzo). Let A be a uniform algebra on a compact Hausdorff space $X$ whose maximal ideal space is $X$ and $E \subset X$ be a closed subset such that $X \backslash E$ is an m-dimensional manifold. Assume that

i. for any $p \in X \backslash E$ there exists $f_{1}, \cdots, f_{m} \in A$ that are $C^{1}$-smooth on $X \backslash E$ and $d f_{1} \wedge \cdots \wedge$ $d f_{m}(p) \neq 0$,

ii. the functions in $A$ that are $C^{1}$-smooth on $X \backslash E$ separate points on $X$.

Then $A=\{g \in C(X): g|E \in A| E\}$.

As pointed out to us by Alexander Izzo, a result along the lines of Theorem 1 (for a similar class of domains) can be obtained from [Izz11] as follows. Let us take $X$ to be the maximal ideal space (spectrum) of $H^{\infty}(\Omega)$ and $X \backslash E$ to be the set of points in $\Omega$ where the Jacobian of $f$ has rank $n$ with $A$ being the closure of $H^{\infty}(\Omega)\left[\overline{f_{1}}, \cdots, \overline{f_{m}}\right]$. Then one obtains Theorem 1 if the set $\Omega_{f}$ is replaced by the set of points where $J_{f}$, the Jacobian of $f$, has rank strictly less than $n$ (usually a larger set than $\Omega_{f}$ ).

Next we will present our generalization of the Axler-Čučković-Rao Theorem to $\mathbb{C}^{n}$, but first we will state the commuting problem for Toeplitz operators.

Let $A^{2}(\Omega)$ denote the space of square integrable holomorphic functions on $\Omega$ and $P: L^{2}(\Omega) \rightarrow$ $A^{2}(\Omega)$ be the Bergman projection, the orthogonal projection onto $A^{2}(\Omega)$. For $g \in L^{\infty}(\Omega)$, the Toeplitz operator $T_{g}: A^{2}(\Omega) \rightarrow A^{2}(\Omega)$ is defined as $T_{g} f=P(g f)$ for all $f \in A^{2}(\Omega)$.

The commuting problem can be stated as follows: Let $\phi$ be a non-constant bounded function on $\Omega$. Determine all $\psi \in L^{\infty}(\Omega)$ such that $\left[T_{\phi}, T_{\psi}\right]=0$.

The commuting problem was solved by Arlen Brown and Paul Halmos on the Hardy space of the unit disc in a famous paper [BH64]. However, on the Bergman space, the problem is still open. Many partial answers has been obtained over the years. To list a few, we refer the reader to [AČ91, ĽR98, AČR00, LT17] for results over the unit disc; to [Zhe98, Le08, Le17] for results over the ball in $\mathbb{C}^{n}$; and to [BL11, CY14, AL16] for results on Fock spaces.

In this paper, we want to highlight the following result of Sheldon Axler, Željko Čučković, and Nagisetti Rao (see [AČR00]).

Theorem (Axler-Čučković-Rao). Let $\Omega$ be a bounded domain in $\mathbb{C}$ and $\phi$ be a nonconstant bounded holomorphic function on $\Omega$. Assume that $\psi$ is a bounded measurable function on $\Omega$ such that $T_{\phi}$ and $T_{\psi}$ commute. Then $\psi$ is holomorphic.

As an application of our results, we get the following generalization of the Axler-ČučkovićRao Theorem.

Corollary 2. Let $\Omega$ be a bounded $L^{\infty}$-pseudoconvex domain in $\mathbb{C}^{n}, g \in L^{\infty}(\Omega)$, and $f_{j} \in H^{\infty}(\Omega)$ for $j=1, \ldots, m$ and $n \leq m$. Assume that the Jacobian of the function $f=\left(f_{1}, \ldots, f_{m}\right): \Omega \rightarrow \mathbb{C}^{m}$ has rank $n$ for some $z \in \Omega$ and $T_{g}$ commutes with $T_{f_{j}}$ for $1 \leq j \leq m$. Then $g$ is holomorphic. 
This paper is organized as follows: The next section contains relevant basic facts and results about $\bar{\partial}$-Koszul complex. Then we will present the proofs of Theorems 1 and 2 , We will finish the paper with the proof of Corollaries 1 and 2 .

\section{THE $\bar{\partial}$-KOSZUl COMPLEX}

Let $\Omega$ be a domain in $\mathbb{C}^{n}$ and $V$ be a vector space of dimension $m$ with a basis $\left\{e_{1}, e_{2}, \ldots, e_{m}\right\}$. We define

$$
\wedge^{r} V=\operatorname{span}\left\{e_{j_{1}} \wedge e_{j_{2}} \wedge \cdots \wedge e_{j_{r}}: j_{1}<j_{2}<\cdots<j_{r}\right\}
$$

and $\Gamma_{(r, s)}^{\infty}=\wedge^{r} V \otimes C L_{(0, s)}^{\infty}(\Omega)$ where $r$ and $s$ are nonnegative integers. We note that throughout the paper we use the convention that $\Gamma_{(r, s)}^{\infty}=\{0\}$ if $r \geq m+1$ or $s \geq n+1$. Finally, $C L_{(0,0)}^{\infty}(\Omega)=$ $C L^{\infty}(\Omega)$.

We define the unbounded operator $\bar{\partial}: \Gamma_{(r, s)}^{\infty} \rightarrow \Gamma_{(r, s+1)}^{\infty}$ as $\bar{\partial}\left(e_{J} \otimes W\right)=e_{J} \otimes \bar{\partial} W$ where $e_{J} \in \wedge^{r} V$ and $W \in C L_{(0, s)}^{\infty}(\Omega)$. The operator $\bar{\partial}$ is defined on

$$
\operatorname{Dom}_{\infty}(\bar{\partial})=\left\{f \in \Gamma_{(r, s)}^{\infty}: \bar{\partial} f \in \Gamma_{(r, s+1)}^{\infty}\right\} .
$$

Let $f=\left(f_{1}, \ldots, f_{m}\right): \Omega \rightarrow \mathbb{C}^{m}$ be a bounded holomorphic mapping. Then for $0 \leq s \leq n$ and $0 \leq r \leq m$ we define the operator

with the following properties:

$$
\mathcal{T}_{f}: \Gamma_{(r+1, s)}^{\infty} \rightarrow \Gamma_{(r, s)}^{\infty}
$$

(1) $\mathcal{T}_{f}\left(e_{j} \otimes W\right)=f_{j} W$,

(2) $\mathcal{T}_{f}(A \wedge B)=\mathcal{T}_{f}(A) \wedge B+(-1)^{|A|_{1}} A \wedge \mathcal{T}_{f} B$ (here $|\cdot|_{1}$ is the order of $A$ in $\cup_{r=0}^{m} \Lambda^{r} V$ ),

(3) $\mathcal{T}_{f} \bar{\partial}=\bar{\partial} \mathcal{T}_{f}$ on $\operatorname{Dom}_{\infty}(\bar{\partial})$ for $0 \leq s \leq n$ and $0 \leq r \leq m$,

(4) $\mathcal{T}_{f} \mathcal{T}_{f}=0$ and $\overline{\partial \partial}=0$.

We note that $\mathcal{T}_{f} W=0$ for $W \in \Gamma_{(0, s)}^{\infty}$ and $0 \leq s \leq n$.

Lemma 1. Let $\Omega$ be a bounded domain in $\mathbb{C}^{n}, 0 \leq s \leq n, 0 \leq r \leq m$, and $f=\left(f_{1}, \ldots, f_{m}\right)$ : $\Omega \rightarrow \mathbb{C}^{m}$ be a bounded holomorphic mapping. Assume that $W \in \Gamma_{(r, s)}^{\infty}$ such that $\operatorname{supp}(W) \subset \Omega$ and $\operatorname{supp}(W) \cap f^{-1}(0)=\varnothing$.

i. If $\mathcal{T}_{f} W=0$, then there exists $Y \in \Gamma_{(r+1, s)}^{\infty}$ such that
a. $\mathcal{T}_{f} Y=W$,
b. $\operatorname{supp}(Y) \subset \Omega$ and $\operatorname{supp}(Y) \cap f^{-1}(0)=\varnothing$.

ii. If $\mathcal{T}_{f} W=0$ and $\bar{\partial} W \in \Gamma_{(r, s+1)}^{\infty}$, then there exists $Y \in \Gamma_{(r+1, s)}^{\infty}$ such that

a. $\bar{\partial} Y \in \Gamma_{(r+1, s+1)}^{\infty}$ and $\mathcal{T}_{f} Y=W$,

b. $\operatorname{supp}(Y) \subset \Omega$ and $\operatorname{supp}(Y) \cap f^{-1}(0)=\varnothing$.

Proof. First let us prove the lemma in case $r=m$. In this case one can show that $\mathcal{T}_{f} W=0$ and $\operatorname{supp}(W) \cap f^{-1}(0)=\varnothing$ imply that $W=0$. So we can choose $Y=0 \in \Gamma_{(m+1, s)}^{\infty}$. For the rest of the proof we will assume that $0 \leq r \leq m-1$. 
Now let us prove i. Let $\chi \in C_{0}^{\infty}(\Omega)$ be a smooth compactly supported cut-off function such that $\chi=1$ on a neighborhood of $\operatorname{supp}(W)$ and $\operatorname{supp}(\chi) \cap f^{-1}(0)=\varnothing$. We define

$$
g_{j}=\frac{\chi \overline{f_{j}}}{\sum_{l=1}^{m}\left|f_{l}\right|^{2}}
$$

and

$$
X=\sum_{j=1}^{m} e_{j} \otimes g_{j} \in \Gamma_{(1,0)}^{\infty} .
$$

Then $g_{j} \in C_{0}^{\infty}(\Omega)$ for $j=1,2, \ldots, m$ and $\mathcal{T}_{f} X=1 \in \Gamma_{(0,0)}^{\infty}$ on the support of $W$ because $\chi=1$ on a neighborhood of $\operatorname{supp}(W)$ and $\sum_{j=1}^{m} f_{j}(z) g_{j}(z)=1$ whenever $\chi(z)=1$.

Let us define $Y=X \wedge W \in \Gamma_{(r+1, s)}^{\infty}$. Then $\operatorname{supp}(Y)$ is a compact subset of $\Omega$ and $\operatorname{supp}(Y) \cap$ $f^{-1}(0)=\varnothing$. Furthermore, $\mathcal{T}_{f} X=1$ on the support of $W$ and

$$
\mathcal{T}_{f} Y=\mathcal{T}_{f}(X) \wedge W-X \wedge \mathcal{T}_{f} W=1 \wedge W=W
$$

because $\mathcal{T}_{f} W=0$.

To prove ii. we observe that, in the proof of i. above, $X$ is smooth compactly supported in $\Omega$. Therefore, if $\bar{\partial} W$ is bounded then so is $\bar{\partial} Y$ as $Y=X \wedge W$.

If $f_{j} \in A^{\infty}(\Omega)$ for $j=1,2, \ldots, m$ in the lemma above, we have the following lemma.

Lemma 2. Let $\Omega$ be a bounded domain in $\mathbb{C}^{n}, V$ be an m-dimensional vector space, and $f_{j} \in A^{\infty}(\Omega)$ for $j=1,2, \ldots, m$. Assume that $W \in \wedge^{r} V \otimes C_{(0, s)}^{\infty}(\bar{\Omega})$ for $0 \leq r \leq m, 0 \leq s \leq n$, and $\operatorname{supp}(W) \cap$ $f^{-1}(0)=\varnothing$ where $f=\left(f_{1}, \ldots, f_{m}\right)$. If $\mathcal{T}_{f} W=0$ then there exists $Y \in \wedge^{r+1} V \otimes C_{(0, s)}^{\infty}(\bar{\Omega})$ such that $\operatorname{supp}(Y) \cap f^{-1}(0)=\varnothing$ and $\mathcal{T}_{f} Y=W$.

Proof. The proof of this lemma is very similar to the proof of Lemma 1 , The only difference is that we choose $\chi \in C^{\infty}(\bar{\Omega})$ be a smooth function such that $\chi=1$ on a neighborhood of $\operatorname{supp}(W)$ and $\operatorname{supp}(\chi) \cap f^{-1}(0)=\varnothing$.

Lemma 3. Let $\Omega$ be a bounded $L^{\infty}$-pseudoconvex domain in $\mathbb{C}^{n}, f=\left(f_{1}, \ldots, f_{m}\right): \Omega \rightarrow \mathbb{C}^{m}$ be a bounded holomorphic mapping, and $W \in \Gamma_{(r, s)}^{\infty}$ for $0 \leq r \leq m$ and $1 \leq s \leq n$ such that

i. $\operatorname{supp}(W) \subset \Omega$ and $\operatorname{supp}(W) \cap f^{-1}(0)=\varnothing$,

ii. $\bar{\partial} W=0$ and $\mathcal{T}_{f} W=0$.

Then there exists $Y \in \Gamma_{(r+1, s-1)}^{\infty}$ such that $Y \in \operatorname{Dom}_{\infty}(\bar{\partial})$ and $\mathcal{T}_{f} \bar{\partial} Y=W$.

Proof. In case $r=m$, as in the proof of Lemma1, one can show that if $W$ satisfies the conditions of the lemma then $W=0$. So we can choose $Y=0$. For the rest of the proof we will assume that $0 \leq r \leq m-1$.

First we will assume that $\Omega$ is a bounded $L^{\infty}$-pseudoconvex domain. We will use a descending induction on $s$ to prove this lemma. So let $s=n, 0 \leq r \leq m-1$, and $W \in \Gamma_{(r, n)}^{\infty}$ such that 
$\operatorname{supp}(W) \subset \Omega, \operatorname{supp}(W) \cap f^{-1}(0)=\varnothing$, and $\mathcal{T}_{f} W=0(\bar{\partial} W=0$ as any $(0, n)$-form is $\bar{\partial}$-closed). Then $i$. in Lemma 1 implies that there exists $Y_{1} \in \Gamma_{(r+1, n)}^{\infty}$ with the following properties:

i. $\operatorname{supp}\left(Y_{1}\right) \subset \Omega$ and $\operatorname{supp}\left(Y_{1}\right) \cap f^{-1}(0)=\varnothing$,

ii. $\mathcal{T}_{f} Y_{1}=W$.

Furthermore, since $Y_{1} \in \Gamma_{(r+1, n)}^{\infty}$ it is $\bar{\partial}$-closed. Then (since $\Omega$ is $L^{\infty}$-pseudoconvex) there exists $Y \in \Gamma_{(r+1, n-1)}^{\infty}$ such that $\bar{\partial} Y=Y_{1}$. That is, $\mathcal{T}_{f} \bar{\partial} Y=W$.

Now we will assume that the lemma is true for $s=k+1, k+2, \ldots, n$ and $r=0,1, \ldots, m-1$. Let $0 \leq r \leq m-1$ and assume that $W \in \Gamma_{(r, k)}^{\infty}$ with the following properties:

i. $\operatorname{supp}(W) \subset \Omega$ and $\operatorname{supp}(W) \cap f^{-1}(0)=\varnothing$,

ii. $\bar{\partial} W=0$ and $\mathcal{T}_{f} W=0$.

Then ii. in Lemma11implies that there exists $Y_{1} \in \Gamma_{(r+1, k)}^{\infty}$ such that

i. $\bar{\partial} Y_{1} \in \Gamma_{(r+1, k+1)}^{\infty}$ and $W=\mathcal{T}_{f} Y_{1}$,

ii. $\operatorname{supp}\left(Y_{1}\right) \subset \Omega$ and $\operatorname{supp}\left(Y_{1}\right) \cap f^{-1}(0)=\varnothing$.

Then

$$
\mathcal{T}_{f} \bar{\partial} Y_{1}=\bar{\partial} \mathcal{T}_{f} Y_{1}=\bar{\partial} W=0 .
$$

So $\bar{\partial} Y_{1}$ satisfies the conditions in the lemma for $s=k+1$. That is, $\bar{\partial} Y_{1} \in \Gamma_{(r+1, k+1)}^{\infty}$ such that

i. $\operatorname{supp}\left(\bar{\partial} Y_{1}\right) \subset \Omega$ and $\operatorname{supp}\left(\bar{\partial} Y_{1}\right) \cap f^{-1}(0)=\varnothing$,

ii. $\overline{\partial \partial} Y_{1}=0$ and $\mathcal{T}_{f} \bar{\partial} Y_{1}=\bar{\partial} W=0$.

By the induction hypothesis, there exists $Y_{2} \in \Gamma_{(r+2, k)}^{\infty}$ such that $\bar{\partial} Y_{2} \in \Gamma_{(r+2, k+1)}^{\infty}$ and $\mathcal{T}_{f} \bar{\partial} Y_{2}=$ $\bar{\partial} Y_{1}$. Then

$$
\bar{\partial} \mathcal{T}_{f} Y_{2}=\mathcal{T}_{f} \bar{\partial} Y_{2}=\bar{\partial} Y_{1}
$$

We define $Y_{3}=Y_{1}-\mathcal{T}_{f} Y_{2} \in \Gamma_{(r+1, k)}^{\infty}$. Then the equality above implies that

$$
\mathcal{T}_{f} Y_{3}=\mathcal{T}_{f} Y_{1}-\mathcal{T}_{f} \mathcal{T}_{f} Y_{2}=W
$$

and $\bar{\partial} Y_{3}=\bar{\partial} Y_{1}-\bar{\partial} \mathcal{T}_{f} Y_{2}=0$. Since $\Omega$ is $L^{\infty}$-pseudoconvex domain we conclude that there exists $Y \in \Gamma_{(r+1, k-1)}^{\infty}$ such that $\bar{\partial} Y=Y_{3}$. That is, $\mathcal{T}_{f} \bar{\partial} Y=W$. Hence the proof of Lemma 3 is complete.

Lemma 4. Let $\Omega$ be a smooth bounded pseudoconvex domain in $\mathbb{C}^{n}, V$ be an m-dimensional vector space, and $f_{i} \in A^{\infty}(\Omega)$ for $i=1, \ldots, m$. Assume that $W \in \wedge^{r} V \otimes C_{(0, s)}^{\infty}(\bar{\Omega})$ for $0 \leq r \leq m$ and $1 \leq s \leq n$ such that $\operatorname{supp}(W) \cap f^{-1}(0)=\varnothing, \bar{\partial} W=0$, and $\mathcal{T}_{f} W=0$. Then there exists $Y \in \wedge^{r+1} V \otimes C_{(0, s-1)}^{\infty}(\bar{\Omega})$ such that $\mathcal{T}_{f} \bar{\partial} Y=W$.

Proof. This proof is similar to the proof of Lemma 3 with the following changes: Instead of Lemma 1 we use Lemma 2 and, at the last step (since and $f_{j} \in A^{\infty}(\Omega)$ ), we use the following result of Joseph Kohn [Koh73] (see also [CS01, Theorem 6.1.1]): Let $\Omega$ be a smooth bounded 
pseudoconvex domain in $\mathbb{C}^{n}, 1 \leq q \leq n$, and $u \in C_{(0, q)}^{\infty}(\bar{\Omega})$ with $\bar{\partial} u=0$. Then there exists $f \in C_{(0, q-1)}^{\infty}(\bar{\Omega})$ such that $\bar{\partial} f=u$.

Lemma 5. Let $\Omega$ be a bounded domain in $\mathbb{C}^{n}$ and $f_{j} \in H^{\infty}(\Omega)$ for $j=1, \ldots$, m such that $\sum_{j=1}^{m}\left|f_{j}\right|^{2}>\varepsilon$ on $\Omega$ for some $\varepsilon>0$ and $\partial f_{j} \in L_{(1,0)}^{\infty}(\Omega)$ for $j=1, \ldots, m$. Assume that $W \in \Gamma_{(r, s)}^{\infty}$ for $0 \leq r \leq m$ and $0 \leq s \leq n$ such that $\mathcal{T}_{f} W=0$ and $\bar{\partial} W \in \Gamma_{(r, s+1)}^{\infty}$. Then there exists $Y \in \Gamma_{(r+1, s)}^{\infty}$ such that $\bar{\partial} Y \in \Gamma_{(r+1, s+1)}^{\infty}$ and $\mathcal{T}_{f} Y=W$.

Proof. The proof will be similar to the proof of Lemma 1. Let $V$ be a vector space of dimension $m$ and $\left\{e_{1}, e_{2}, \ldots, e_{m}\right\}$ be a basis for $V$. We define

$$
g_{j}=\frac{\overline{f_{j}}}{\sum_{l=1}^{m}\left|f_{l}\right|^{2}}
$$

and $X=\sum_{j=1}^{m} e_{j} \otimes g_{j} \in \Gamma_{(1,0)}^{\infty}$. Then $g_{j} \in L^{\infty}(\Omega)$ and

$$
\bar{\partial} g_{j}=\frac{\overline{\partial f_{j}}}{\sum_{l=1}^{m}\left|f_{l}\right|^{2}}-\frac{\overline{f_{j}} \sum_{l=1}^{m} f_{l} \overline{\partial f_{l}}}{\left(\sum_{l=1}^{m}\left|f_{l}\right|^{2}\right)^{2}} \in L_{(0,1)}^{\infty}(\Omega) .
$$

Furthermore, $\bar{\partial} X=\sum_{j=1}^{m} e_{j} \otimes \bar{\partial} g_{j} \in \Gamma_{(1,1)}^{\infty}$. Then $Y=X \wedge W \in \Gamma_{(r+1, s)}^{\infty}$ satisfies the following properties: $\bar{\partial} Y=\bar{\partial} X \wedge W+X \wedge \bar{\partial} W \in \Gamma_{(r+1, s+1)}^{\infty}$ and

$$
\mathcal{T}_{f} Y=\mathcal{T}_{f}(X) \wedge W-X \wedge \mathcal{T}_{f} W=1 \wedge W=W
$$

as $\mathcal{T}_{f} W=0$.

Proposition 1. Let $\Omega$ be a bounded $L^{\infty}$-pseudoconvex domain in $\mathbb{C}^{n}$ and $f_{j} \in H^{\infty}(\Omega)$ for $j=1, \ldots, m$ such that $\sum_{j=1}^{m}\left|f_{j}\right|^{2}>\varepsilon$ on $\Omega$ for some $\varepsilon>0$ and $\partial f_{j} \in L_{(1,0)}^{\infty}(\Omega)$ for $j=1, \ldots, m$. Assume that $W \in \Gamma_{(r, s)}^{\infty}$ for $0 \leq r \leq m$ and $0 \leq s \leq n$ such that $\bar{\partial} W=0$ and $\mathcal{T}_{f} W=0$. Then there exists $Y \in \Gamma_{(r+1, s)}^{\infty}$ such that $\bar{\partial} Y=0$ and $\mathcal{T}_{f} Y=W$.

Proof. We will use a descending induction on $s$ as in the proof of Proposition 1, Let $s=n$. Any form of type $(r, n)$ for $0 \leq r \leq m$ is $\bar{\partial}$-closed. Then $\bar{\partial} Y=0$ and Lemma 5 implies that there exists $Y \in \Gamma_{(r+1, n)}^{\infty}$ such that $\mathcal{T}_{f} Y=W$.

Now we will assume that the lemma is true for $s=l+1, l+2, \ldots, n$ and $r=0,1, \ldots, m$ to prove that it is also true for $s=l \leq n-1$ and $0 \leq r \leq m$.

Assume that $W \in \Gamma_{(r, l)}^{\infty}$ such that $\bar{\partial} W=0$ and $\mathcal{T}_{f} W=0$. Then Lemma 5 implies that there exists $\widetilde{Y} \in \Gamma_{(r+1, l)}^{\infty}$ such that $\bar{\partial} \widetilde{Y} \in \Gamma_{(r+1, l+1)}^{\infty}$ and $W=\mathcal{T}_{f} \widetilde{Y}$. Then

$$
\mathcal{T}_{f} \bar{\partial} \widetilde{Y}=\bar{\partial} \mathcal{T}_{f} \widetilde{Y}=\bar{\partial} W=0 .
$$

So $\bar{\partial} \widetilde{Y}$ satisfies the conditions in the lemma for $s=l+1$. That is, $\bar{\partial} \widetilde{Y} \in \Gamma_{(r+1, l+1)}^{\infty}, \overline{\partial \partial} \widetilde{Y}=0$ and $\mathcal{T}_{f} \bar{\partial} \widetilde{Y}=\bar{\partial} W=0$. Then, by the induction hypothesis, there exists $Y_{1} \in \Gamma_{(r+2, l+1)}^{\infty}$ such that $\bar{\partial} Y_{1}=0$ 
and $\mathcal{T}_{f} Y_{1}=\bar{\partial} \widetilde{Y}$. Then since $\Omega$ is a $L^{\infty}$-pseudoconvex domain there exists $Y_{2} \in \Gamma_{(r+2, l)}^{\infty}$ such that $\bar{\partial} Y_{2}=Y_{1}$. Then

$$
\bar{\partial} \mathcal{T}_{f} Y_{2}=\mathcal{T}_{f} \bar{\partial} Y_{2}=\mathcal{T}_{f} Y_{1}=\bar{\partial} \widetilde{Y} .
$$

We define $Y=\widetilde{Y}-\mathcal{T}_{f} Y_{2} \in \Gamma_{(r+1, l)}^{\infty}$. Then the equality above implies that $\bar{\partial} Y=\bar{\partial} \widetilde{Y}-\bar{\partial} \mathcal{T}_{f} Y_{2}=0$ and

$$
\mathcal{T}_{f} Y=\mathcal{T}_{f} \widetilde{Y}-\mathcal{T}_{f} \mathcal{T}_{f} Y_{2}=W
$$

Hence the proof of Proposition 1 is complete.

As a corollary to the previous proposition (with $W=1$ and $r=s=0$ ) we get the following Corona type result. We refer the reader to [Kra14] and the references therein for more information about Corona problem on domains in $\mathbb{C}^{n}$.

Corollary 3. Let $\Omega$ be a bounded $L^{\infty}$-pseudoconvex domain in $\mathbb{C}^{n}$ and $f_{j} \in H^{\infty}(\Omega)$ for $j=1, \ldots, m$ such that $\sum_{j=1}^{m}\left|f_{j}\right|^{2}>\varepsilon$ on $\Omega$ for some $\varepsilon>0$ and $\partial f_{j} \in L_{(1,0)}^{\infty}(\Omega)$ for $j=1, \ldots, m$. Then there exists $g_{i} \in H^{\infty}(\Omega)$ for $j=1, \ldots, m$ such that $\sum_{j=1}^{m} f_{j} g_{j}=1$.

\section{PROOFS OF RESULTS}

The proofs of the theorems are mainly inspired by the proof in Christopher Bishop's paper [Bis89].

Proofs of Theorems 1 1and 2. The proofs of both theorems are very similar. So we will present the proof of Theorem 1 and comment on how the proof of Theorem 2 differs as we go along.

Let $\epsilon>0$ and $\lambda \in \mathbb{C}^{m}$. Since $g \in C(\bar{\Omega})$ and $\left.g\right|_{b \Omega \cup \Omega_{f}}=0$, there exist $g^{\lambda} \in C^{\infty}(\bar{\Omega})$ such that

i. $\sup \left\{\left|g(z)-g^{\lambda}(z)\right|: z \in \bar{\Omega}\right\}<\varepsilon$,

ii $\operatorname{supp}\left(\bar{\partial} g^{\lambda}\right) \cap\left(b \Omega \cup f^{-1}(\lambda)\right)=\varnothing$.

In the proof Theorem 2 the second condition above is replaced by $\operatorname{supp}\left(\bar{\partial} g^{\lambda}\right) \cap f^{-1}(\lambda)=\varnothing$. This can be seen as follows: We choose an open set $U_{\varepsilon}$ in $\mathbb{C}^{n}$ containing $f^{-1}(\lambda)$ and $g_{\varepsilon} \in A^{\infty}\left(U_{\varepsilon} \cap \Omega\right)$ such that $\left|g-g_{\varepsilon}\right|<\varepsilon / 2$ on $f^{-1}(\lambda)$. Then we choose $\chi_{\varepsilon} \in C_{0}^{\infty}\left(U_{\varepsilon}\right)$ such that, $0 \leq \chi_{\varepsilon} \leq 1, \chi_{\varepsilon}=1$ on a neighborhood of $f^{-1}(\lambda)$, and

$$
\operatorname{supp}\left(\chi_{\varepsilon}\right) \cap \bar{\Omega} \subset\left\{z \in U_{\varepsilon} \cap \bar{\Omega}:\left|g(z)-g_{\varepsilon}(z)\right|<\varepsilon\right\} .
$$

Then we define $g^{\lambda}=\left(1-\chi_{\varepsilon}\right) g+\chi_{\varepsilon} g_{\varepsilon}$. Since $g^{\lambda}$ is holomorphic on a neighborhood of $f^{-1}(\lambda)$ we have $\bar{\partial} g^{\lambda}=0$ on the same neighborhood. Furthermore, $\left|g^{\lambda}(z)-g(z)\right|=\chi_{\varepsilon}(z)\left|g_{\varepsilon}(z)-g(z)\right|<\varepsilon$ for all $z \in \bar{\Omega}$.

Using Lemma 3 with $r=0, s=1$, and $W=\bar{\partial} g^{\lambda}$ we get $Y=\sum_{l=1}^{m} e_{l} \otimes H_{l} \in \Gamma_{(1,0)}^{\infty}$ such that

$$
\bar{\partial} g^{\lambda}=\mathcal{T}_{f-\lambda} \bar{\partial} Y=\sum_{l=1}^{m}\left(f_{l}-\lambda_{l}\right) \bar{\partial} H_{l}^{\lambda} .
$$


The above equality implies that

$$
G_{\lambda}=g^{\lambda}-\sum_{l=1}^{m}\left(f_{l}-\lambda_{l}\right) H_{l}^{\lambda}
$$

is a bounded holomorphic function.

In the proof of Theorem 2 , we use Lemma 4 and get $H_{l}^{\lambda} \in C^{\infty}(\bar{\Omega})$ for $l=1, \ldots, m$ in the equation (1) and $G_{\lambda}$ is smooth up to the boundary. Therefore, for $z \in \Omega$ we have

$$
\left|G_{\lambda}(z)-g^{\lambda}(z)\right| \leq \sum_{l=1}^{m}\left|f_{l}(z)-\lambda_{l}\right| \sum_{s=1}^{m}\left|H_{s}^{\lambda}(z)\right| .
$$

Then the above inequality implies that for $M_{\lambda}=\sum_{s=1}^{m}\left\|H_{s}^{\lambda}\right\|_{L^{\infty}(\Omega)}<\infty$ we have

$$
\left|G_{\lambda}(z)-g^{\lambda}(z)\right| \leq M_{\lambda}|f(z)-\lambda|
$$

for $z \in \Omega$.

Compactness of $\overline{f(\Omega)}$ implies that we can choose a finite collection of points $\left\{\lambda_{j}\right\}_{j=1}^{k} \subset \overline{f(\Omega)}$ such that $\left\{B\left(\lambda^{j}, \epsilon M_{\lambda^{j}}^{-1}\right)\right\}_{j=1}^{k}$ forms a finite open cover for $\overline{f(\Omega)}$. Let $\left\{\chi_{j}\right\}_{j=1}^{k}$ be a smooth partition of unity on $\overline{f(\Omega)}$ such that $0 \leq \chi_{j} \leq 1$ and $\operatorname{supp}\left(\chi_{j}\right) \subset U_{j}$. Then $\left\{f^{-1}\left(B\left(\lambda^{j}, \epsilon M_{\lambda^{j}}^{-1}\right)\right)\right\}_{j=1}^{k}$ is an cover for $\Omega$ and $\left|f(z)-\lambda^{j}\right|<\epsilon M_{\lambda^{j}}^{-1}$ for $z \in f^{-1}\left(B\left(\lambda^{j}, \epsilon M_{\lambda^{j}}^{-1}\right)\right)$. Then for $z \in \Omega$ we have

$$
\begin{aligned}
\left|\sum_{j=1}^{k} G_{\lambda^{j}}(z) \chi_{j}(f)(z)-g(z)\right| & \leq \sum_{j=1}^{k}\left|G_{\lambda^{j}}(z)-g(z)\right| \chi_{j}(f(z)) \\
& \leq \sum_{j=1}^{k}\left|G_{\lambda^{j}}(z)-g^{\lambda^{j}}\right| \chi_{j}(f(z))+\sum_{j=1}^{k}\left|g^{\lambda^{j}}(z)-g(z)\right| \chi_{j}(f(z)) \\
& \leq \sum_{j=1}^{k} M_{\lambda^{j}}\left|f(z)-\lambda^{j}\right| \chi_{j}(f(z))+\varepsilon \sum_{j=1}^{k} \chi_{j}(f(z)) \\
& \leq 2 \epsilon .
\end{aligned}
$$

Finally, the Stone-Weierstrass Theorem implies that $\chi_{j}(f)$ can be approximated uniformly on $\bar{\Omega}$ by elements of $\mathbb{C}\left[f_{1}, \ldots, f_{m}, \overline{f_{1}}, \ldots, \overline{f_{m}}\right]$. Hence the proofs of Theorems 1 and 2 are complete.

Hartogs Extension Theorem together Theorem 2 lead to the following corollary.

Corollary 4. Let $\Omega$ be a bounded $L^{\infty}$-pseudoconvex domain in $\mathbb{C}^{n}$. Assume that $f=\left(f_{1}, \ldots, f_{m}\right)$ : $\Omega \rightarrow \mathbb{C}^{m}$ be a bounded holomorphic mapping and $g \in C(\bar{\Omega})$ such that $\bar{\partial} g$ is supported away from $b \Omega$ and the set of points at which the Jacobian of $f$ has rank strictly less than $n$. Then $g$ belongs to the closure of $H^{\infty}(\Omega)\left[\overline{f_{1}}, \cdots, \overline{f_{m}}\right]$ in $L^{\infty}(\Omega)$.

Proof. Since $\bar{\partial} g$ vanishes near the boundary of $\Omega$, Hartogs Extension Theorem implies that there exists $g_{1} \in H^{\infty}(\Omega)$ such that $g=g_{1}$ near the boundary of $\Omega$. Then $g_{2}=g-g_{1} \in C(\bar{\Omega})$ and $g_{2}$ is compactly supported in $\Omega$. Furthermore, $g_{2}$ is holomorphic on a neighborhood of the set 
where the Jacobian of $f$ has rank strictly less than $n$. Therefore, Theorem 2 implies that $g_{2}$ can be approximated in the sup-norm by functions in $H^{\infty}(\Omega)\left[\overline{f_{1}}, \ldots, \overline{f_{m}}\right]$. This completes the proof of the corollary.

Next we provide the proof of Corollary 1 .

Proof of Corollary 1. Obviously i. implies ii. So to prove that ii. implies iii., let us assume that $H^{\infty}(\Omega)\left[\overline{f_{1}}, \ldots, \overline{f_{m}}\right]$ is dense in $L^{p}(\Omega)$ for some $1 \leq p<\infty$. Let $B \subset \Omega$ be a ball such that $\bar{B} \subset \Omega$. Then, the algebra $H^{\infty}(B)\left[\overline{f_{1}}, \ldots, \overline{f_{m}}\right]$ is dense in $L^{p}(B)$ for some $1 \leq p<\infty$. Moreover, the algebra generated by $\left\{z_{1}, \ldots, z_{n}\right\}$ is dense in $H^{\infty}(B)$ and $f_{1}, \cdots, f_{m}$ are holomorphic on a neighborhood of $\bar{B}$. Next we adopt [IL13, Theorem 4.2] to our set-up. Namely, [IL13, Theorem 4.2] implies that if the algebra generated by $\left\{z_{1}, \ldots, z_{n}, \bar{f}_{1}, \ldots, \bar{f}_{m}\right\} \subset C^{\infty}(B)$ is dense in $L^{p}(B)$ for some $1 \leq p<\infty$ then the real Jacobian of $\left\{z_{1}, \ldots, z_{n}, \bar{f}_{1}, \ldots, \bar{f}_{m}\right\}$ is of full rank on a dense open set in $B$. Hence the rank of $J_{f}$ is $n$ on a dense open subset in $B$ and (by identity principle) in $\Omega$. Hence, we have iii.

Finally, to prove iii. implies i. we assume that the rank of $J_{f}$ is $n$ for some $z \in \Omega$. Then, the set of points at which $J_{f}$ has rank strictly less than $n$ is a closed set of measure 0 (see [Ran86, Theorem 3.7]). One can show that $X_{f}$, the set of smooth functions with compact support in $\Omega$ and vanish where $J_{f}$ has rank strictly less than $n$, is dense in $L^{p}(\Omega)$ for all $0<p<\infty$. On the other hand, Theorem 1 implies that any function in $X_{f}$ is in the closure of $H^{\infty}(\Omega)\left[\overline{f_{1}}, \ldots, \overline{f_{m}}\right]$ in $L^{\infty}(\Omega)$. Therefore, $H^{\infty}(\Omega)\left[\overline{f_{1}}, \ldots, \overline{f_{m}}\right]$ is dense in $L^{p}(\Omega)$. Hence, we have i.

We finally end the paper with the proof of Corollary 2.

Proof of Corollary 2. We will use the fact that $T_{g}$ can be defined by the following formula

$$
\left\langle T_{g} \phi, \psi\right\rangle_{A^{2}(\Omega)}=\langle g \phi, \psi\rangle_{L^{2}(\Omega)}
$$

for all $\phi, \psi \in A^{2}(\Omega)$. Since $T_{g}$ commutes with $T_{P(f)}$, for any holomorphic polynomial $P$, we have

$$
\langle g P(f), \psi\rangle=\left\langle T_{g} T_{P(f)}(1), \psi\right\rangle=\left\langle P(f) T_{g}(1), \psi\right\rangle
$$

for all $\psi \in A^{2}(\Omega)$. Then $\left\langle T_{g}(1)-g, \overline{P(f)} \psi\right\rangle=0$ for all $\psi \in A^{2}(\Omega)$. Since, by Corollary 1 , the subspace generated by $\left\{\overline{P(f)} \psi: \psi \in A^{2}(\Omega)\right\}$ is dense in $L^{2}(\Omega)$, we conclude that $T_{g}(1)=g$. That is, $g$ is holomorphic.

\section{ACKNOWLEDGEMENT}

We would like to thank Alexander Izzo for reading an earlier manuscript of this paper and for providing us with valuable comments. We are also thankful to the anonymous referee for helpful feedback. 


\section{REFERENCES}

[AČ91] Sheldon Axler and Željko Čučković, Commuting Toeplitz operators with harmonic symbols, Integral Equations Operator Theory 14 (1991), no. 1, 1-12.

[AČR00] Sheldon Axler, Željko Čučković, and N. V. Rao, Commutants of analytic Toeplitz operators on the Bergman space, Proc. Amer. Math. Soc. 128 (2000), no. 7, 1951-1953.

[AL16] Amila Appuhamy and Trieu Le, Commutants of Toeplitz operators with separately radial polynomial symbols, Complex Anal. Oper. Theory 10 (2016), no. 1, 1-12.

[AS87] Sheldon Axler and Allen Shields, Algebras generated by analytic and harmonic functions, Indiana Univ. Math. J. 36 (1987), no. 3, 631-638.

[BH64] Arlen Brown and P. R. Halmos, Algebraic properties of Toeplitz operators, J. Reine Angew. Math. 213 (1963/1964), 89-102.

[Bis89] Christopher J. Bishop, Approximating continuous functions by holomorphic and harmonic functions, Trans. Amer. Math. Soc. 311 (1989), no. 2, 781-811.

[BL11] Wolfram Bauer and Trieu Le, Algebraic properties and the finite rank problem for Toeplitz operators on the SegalBargmann space, J. Funct. Anal. 261 (2011), no. 9, 2617-2640.

[Cao08] Guangfu Cao, On a problem of Axler, Cuckovic and Rao, Proc. Amer. Math. Soc. 136 (2008), no. 3, 931-935 (electronic).

[ČR98] Željko Čučković and N. V. Rao, Mellin transform, monomial symbols, and commuting Toeplitz operators, J. Funct. Anal. 154 (1998), no. 1, 195-214.

[CS01] So-Chin Chen and Mei-Chi Shaw, Partial differential equations in several complex variables, AMS/IP Studies in Advanced Mathematics, vol. 19, American Mathematical Society, Providence, RI; International Press, Boston, MA, 2001.

[CY14] Boo Rim Choe and Jongho Yang, Commutants of Toeplitz operators with radial symbols on the Fock-Sobolev space, J. Math. Anal. Appl. 415 (2014), no. 2, 779-790.

[DFF99] Klas Diederich, Bert Fischer, and John Erik Fornæss, Hölder estimates on convex domains of finite type, Math. Z. 232 (1999), no. 1, 43-61.

[FLZ11] John Erik Fornæss, Lina Lee, and Yuan Zhang, On supnorm estimates for $\bar{\partial}$ on infinite type convex domains in $\mathbb{C}^{2}$, J. Geom. Anal. 21 (2011), no. 3, 495-512.

[IL13] Alexander J. Izzo and Bo Li, Generators for algebras dense in $L^{p}$-spaces, Studia Math. 217 (2013), no. 3, 243263.

[Izz11] Alexander J. Izzo, Uniform approximation on manifolds, Ann. of Math. (2) 174 (2011), no. 1, 55-73.

[Koh73] J. J. Kohn, Global regularity for $\bar{\partial}$ on weakly pseudo-convex manifolds, Trans. Amer. Math. Soc. 181 (1973), 273-292.

[Kra14] Steven G. Krantz, The corona problem in several complex variables, The corona problem, Fields Inst. Commun., vol. 72, Springer, New York, 2014, pp. 107-126.

[Le08] Trieu Le, The commutants of certain Toeplitz operators on weighted Bergman spaces, J. Math. Anal. Appl. 348 (2008), no. 1, 1-11.

[Le17] Commutants of separately radial Toeplitz operators in several variables, J. Math. Anal. Appl. 453 (2017), no. 1, 48-63.

[LT17] Trieu Le and Akaki Tikaradze, Commutants of Toeplitz operators with harmonic symbols, New York J. Math. 23 (2017), 1723-1731.

[Ran86] R. Michael Range, Holomorphic functions and integral representations in several complex variables, Graduate Texts in Mathematics, vol. 108, Springer-Verlag, New York, 1986. 
[Ran90] _ Integral kernels and Hölder estimates for $\bar{\partial}$ on pseudoconvex domains of finite type in $\mathbb{C}^{2}$, Math. Ann. 288 (1990), no. 1, 63-74.

[SH80] A. G. Sergeev and G. M. Henkin, Uniform estimates of the solutions of the $\bar{\partial}$-equation in pseudoconvex polyhedra, Mat. Sb. (N.S.) 112(154) (1980), no. 4(8), 522-567, translation in Math. USSR-Sb. 40 (1981), no. 4, 469-507.

[SW12] Håkan Samuelsson and Erlend Fornæss Wold, Uniform algebras and approximation on manifolds, Invent. Math. 188 (2012), no. 3, 505-523.

[Zhe98] Dechao Zheng, Commuting Toeplitz operators with pluriharmonic symbols, Trans. Amer. Math. Soc. 350 (1998), no. 4, 1595-1618.

E-mail address: Sonmez.Sahutoglu@utoledo.edu, Akaki.Tikaradze@utoledo.edu

University of Toledo, Department of MATHEMATics \& StATistics, TOledo, OH 43606, USA 